\title{
Zero- to ultralow-field NMR spectroscopy of
}

\section{small biomolecules}

\section{Supplementary Information}

Piotr Put, ${ }^{\dagger}$ Szymon Pustelny, ${ }^{\dagger}$ Dmitry Budker,,$\uparrow^{\ddagger}$ Emanuel Druga, ${ }^{\S}$ Tobias F. Sjolander,,$^{\S}$ Alexander Pines,, and Danila A. Barskiy ${ }^{*, \ldots, \S}$

$\dagger$ M. Smoluchowski Institute of Physics, Jagiellonian University, Eojasiewicza 11, 30-348 Kraków, Poland

$\ddagger$ Helmholtz-Institut Mainz, Johannes Gutenberg-Universität, Staudingerweg 18, 55128 Mainz, 55099, Mainz, Germany

\Department of Physics, University of California at Berkeley, Berkeley, California 94720-7300, USA

$\S$ Department of Chemistry, University of California at Berkeley, Berkeley, California 94720-1462, USA

E-mail: dbarskiy@uni-mainz.de

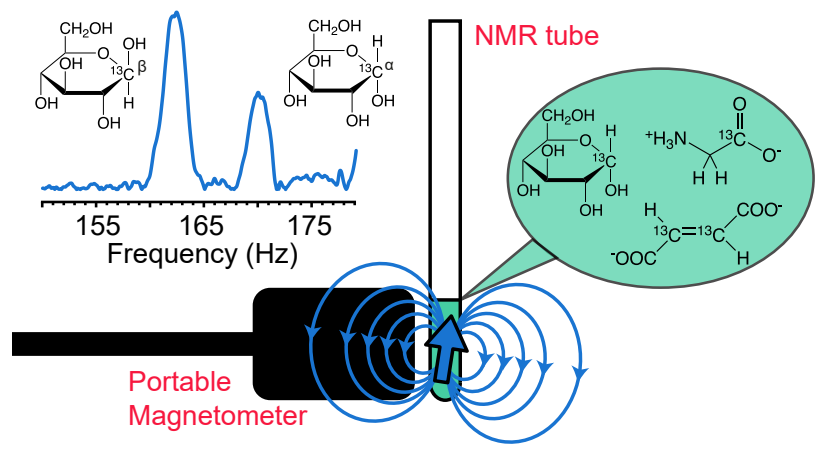




\section{DC pulse calibration}

Low-frequency Larmor precession experiments can be used to calibrate magnetic-field pulses. A small magnetic field was applied with a shimming coil along the guiding-field direction. Upon an adiabatic turn off of the guiding field, no precession was observed. Then, a DC magnetic-field pulse was applied in one of the perpendicular directions, tipping the spins away from their initial direction and a precision signal was recorded. Since the amplitude of the measured signal follows the $\left|\cos \left(\gamma B_{D C} \tau\right)\right|$-dependence, where $B_{D C}$ is the amplitude of the magnetic pulse, $\tau$ is its length, and $\gamma$ is the gyromagnetic ratio of the measured nuclei, to obtain the pulse calibration, the pulse length/strength was varied and the calibration curve was reconstructed. By changing a direction of a guiding field and pulses, the calibration of all coils was achieved (Fig. S1).
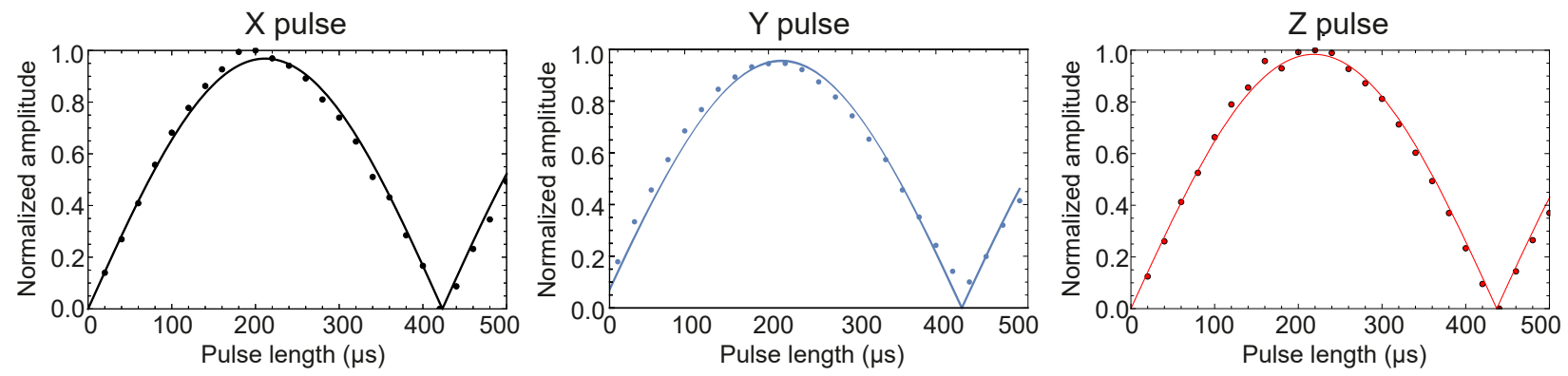

Figure S1: Calibration of DC magnetic-field pulses. The calibration was performed using a NMR signal of water measured at ultra-low magnetic field (50 nT) for three spatial directions.

\section{Magnetic field shimming}

To operate the spectrometer under zero-field conditions not only the field experienced by the sensor needs to be nullified (using small coils built into the sensor) to ensure optimal operation, but it has to be zeroed also for the NMR sample to avoid splitting and broadening of spectral lines. A residual magnetic field inside a degaussed magnetic shielding can exceed $1 \mathrm{nT}$. The shimming procedure was adapted from previous works and works as follows. The near-zero-field spectra of $\left[{ }^{13} \mathrm{C}\right]$-formic acid was recorded. A typically spectrum split into 
three peaks because of the residual fields. The field along initial polarization (along $z$ ) was then zeroed out using the shimming coil, until the central peak disappears. Then both the $x$ and $y$ fields were varied until the split lines merge together at the center (Fig. S2).

(a)

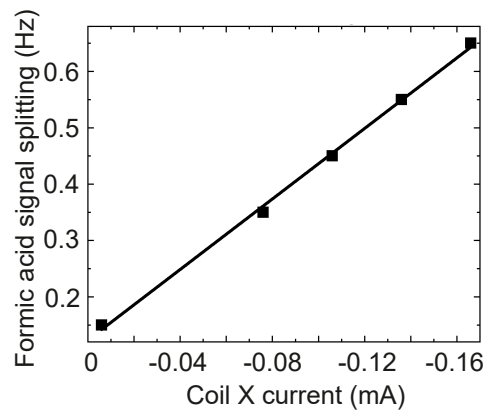

(b)

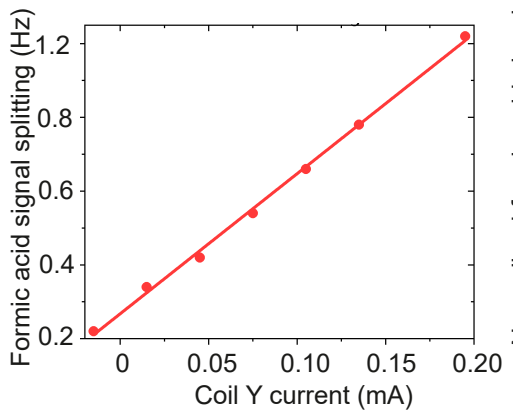

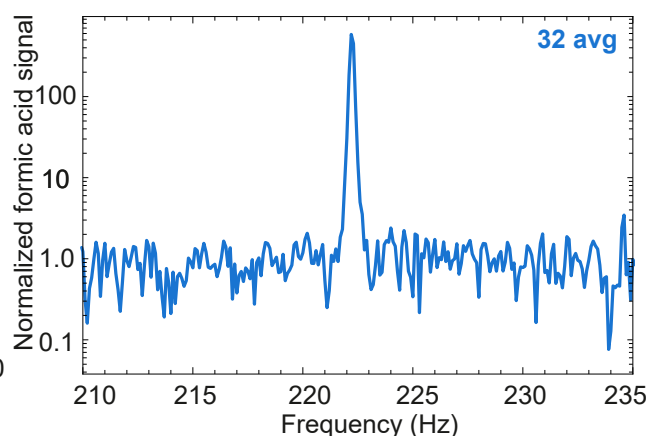

Figure S2: (a) $\left[{ }^{13} \mathrm{C}\right]$-formic acid signal splitting plotted for different fields applied in transverse directions. The splitting is a linear function of the applied field (provided that other transverse field component is negligibly small). This allows for the calibration of the shimming coils and obtaining truly zero-field spectra (Fig. 1). (b) The resultant $\left[{ }^{13} \mathrm{C}\right]$-formic acid spectrum, following $4 \pi$ pulse for protons, obtained by averaging 32 transients. The signal-to-noise ratio in a single transient reaches $\sim 100$.

\section{Samples and preparation}

Samples for the ZULF measurements were prepared in standard 5-mm 600-MHz NMR tubes. The liquid samples were transferred directly to the tube, while the solid samples were dissolved in deionized water. The samples were degaussed by freezing the liquid in either mixture of dry ice and acetone or liquid nitrogen and connecting the tube via a Young valve manifold to vacuum. The cycle or freezing, pumping the gas and thawing were typically repeated 4 times, until the gas pressure over frozen liquid felt below $10^{-4}$ mbar.

All the chemicals used in this work were purchased from Sigma Aldrich (Merck): [1${ }^{13} \mathrm{C}$-glycine (CAS Number 20110-59-2), $\left[2,3-{ }^{13} \mathrm{C}_{2}\right]$-fumaric acid (CAS Number 123548-45-8), $\left[1-{ }^{13} \mathrm{C}\right]$-D-glucose (CAS Number 40762-22-9), D-glucose (CAS number 50-99-7), $\left[{ }^{13} \mathrm{C}\right]$-formic acid (CAS Number 1633-56-3). 


\section{$J$-coupling values}

To support $J$-spectra presented in the article the best matching $J$-coupling values from the zero-field measurement were extracted and compared (if possible) with coupling values reported in the literature (Tab. S1). Overall, the coupling strengths obtained from zero-field spectra match the literature value, while small discrepancies can be explained by a slightly different chemical structure (see fumarate coupling values).

Table S1: J-coupling values measured using zero-field NMR spectroscopy technique in this work and corresponding coupling strengths from the literature. Literature coupling values for fumarate are taken from study of asymmetric fumarate diester.

\begin{tabular}{l||l||l} 
Compound & Measured $J$-coupling $(\mathrm{Hz})$ & $J_{\text {-coupling from the literature }(\mathrm{Hz})}$ \\
\hline \hline$\left[1-{ }^{13} \mathrm{C}\right]$-formic acid & $J_{\mathrm{CH}}=222.2$ & $J_{\mathrm{CH}}=222.2^{1}$ \\
\hline$\left[1-{ }^{13} \mathrm{C}\right]$-glycine & $J_{\mathrm{CH}}=5.2$ & $J_{\mathrm{CH}}=-$ \\
\hline \multirow{2}{*}{$\left.{ }^{13} \mathrm{C}\right]$-D-glucose } & $J_{\mathrm{C}_{\alpha} \mathrm{H}}=170$ & $J_{\mathrm{C}_{\alpha} \mathrm{H}}=169.5^{2}$ \\
& $J_{\mathrm{C}_{\beta} \mathrm{H}}=162$ & $J_{\mathrm{C}_{\beta} \mathrm{H}}=161.3^{2}$ \\
\hline \multirow{2}{*}[2,3-{}^{13}\mathrm{C}_{2}]{-fumarate } & $J_{\mathrm{CH}}=159.3$ & $J_{\mathrm{CH}}=166.7^{3}$ \\
& $J_{\mathrm{CC}}=71.0$ & $J_{\mathrm{CC}}=71.0^{3}$ \\
& $J_{\mathrm{HH}}=15.7$ & $J_{\mathrm{HH}}=15.7^{3}$ \\
& $J_{\mathrm{CH}(2)}=-1.3$ & $J_{\mathrm{CH}(2)}=-2.8^{3}$
\end{tabular}

\section{Experimental apparatus}

Details of the experimental setup and its operation are explained in the article. Here we present a photograph of an exemplary zero-field NMR spectrometer used in this study, showcasing its modularity and mobility (Fig. S3). 


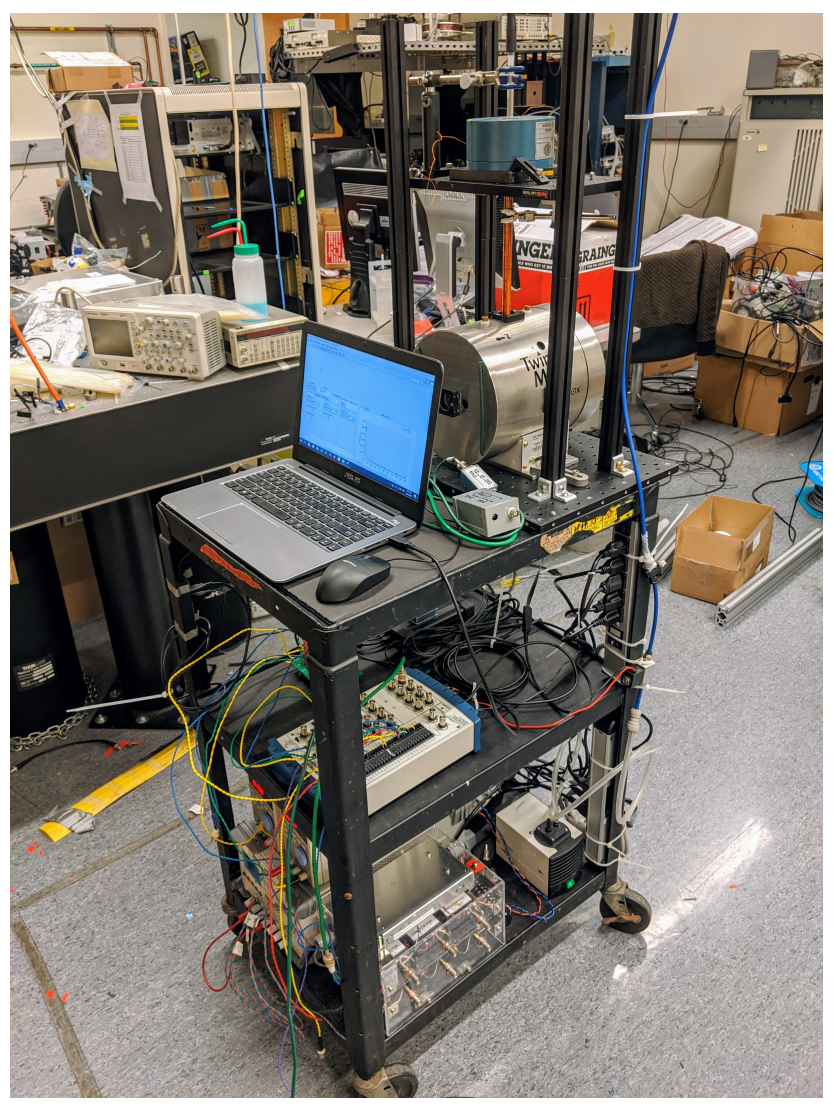

Figure S3: Picture of the portable, modular ZULF NMR spectrometer used in this work. The whole setup was placed on the movable cart. The zero-field magnetic region is enclosed by the magnetic shielding (metallic cylinder behind the laptop screen). The sample was prepolarized with a pernament magnet assembly placed outside (above) of the shield and shuttled to the the zero-field region for detection. The lower parts of the cart house the offthe-shelf electronic components: QuSpin sensor driver, DAQ module, power supplies, relays, current source and compact vacuum pump.

\section{References}

(1) Blanchard, J. W.; Wu, T.; Eills, J.; Hu, Y.; Budker, D. Zero-to ultralow-field nuclear magnetic resonance J-spectroscopy with commercial atomic magnetometers. Journal of Magnetic Resonance 2020, 106723.

(2) Tiainen, M.; Maaheimo, H.; Soininen, P.; Laatikainen, R. 13C isotope effects on 1H chemical shifts: NMR spectral analysis of 13C-labelled D-glucose and some 13C-labelled amino acids. Magnetic Resonance in Chemistry 2010, 48, 117-122. 
(3) Stevanato, G.; Roy, S. S.; Hill-Cousins, J.; Kuprov, I.; Brown, L. J.; Brown, R. C.; Pileio, G.; Levitt, M. H. Long-lived nuclear spin states far from magnetic equivalence. Physical Chemistry Chemical Physics 2015, 17, 5913-5922. 